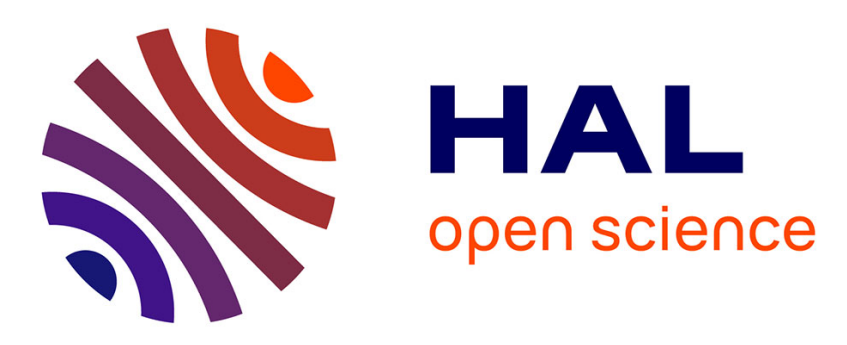

\title{
Self-Fuzzification Method according to Typicality Correlation for Classification on tiny Data Sets
} Emmanuel Schmitt, Vincent Bombardier, Patrick Charpentier

\section{To cite this version:}

Emmanuel Schmitt, Vincent Bombardier, Patrick Charpentier. Self-Fuzzification Method according to Typicality Correlation for Classification on tiny Data Sets. 16th International Conference on Fuzzy Systems, FUZZIEEE'07, Jul 2007, Londres, United Kingdom. pp.1072-1077. hal-00137985

\section{HAL Id: hal-00137985 \\ https://hal.science/hal-00137985}

Submitted on 7 Jan 2008

HAL is a multi-disciplinary open access archive for the deposit and dissemination of scientific research documents, whether they are published or not. The documents may come from teaching and research institutions in France or abroad, or from public or private research centers.
L'archive ouverte pluridisciplinaire HAL, est destinée au dépôt et à la diffusion de documents scientifiques de niveau recherche, publiés ou non, émanant des établissements d'enseignement et de recherche français ou étrangers, des laboratoires publics ou privés. 


\title{
Self-Fuzzification Method according to Typicality Correlation for Classification on tiny Data Sets
}

\author{
Emmanuel Schmitt, Vincent Bombardier and Patrick Charpentier
}

\begin{abstract}
This article presents a self-fuzzification method to enhance the settings of a Fuzzy Reasoning Classification adapted to the automated inspection of wooden boards. The supervised classification is made thanks to fuzzy linguistic rules generated from small training data sets. This study especially answers to a double industrial need about the pattern recognition in wooden boards. Firstly, few samples are available to generate the recognition model. This aspect makes lesser efficient compilation methods like neural networks in terms of recognition rates. Secondly, the settings of the classification method must be simplified, because the users are not experts in fuzzy logic. In this article, two points are presented. The first part demonstrates the generalization capability of the presented classification method in comparison to more classical algorithms. In the second part, we propose a new automatic method of parameter fuzzification, by using the typicality correlation coefficients of each class.
\end{abstract}

\section{INTRODUCTION}

The wooden manufacturing industries are placed in a highly competitive market. They express the need of automated vision systems more and more adapted to the wood quality control (defect detection, color matching). In this field, the processes are generally made by human operators. These people own all the knowledge and the competencies required to make this quality control. That's why, it is important to integrate this knowledge in the vision system in the aim to specify the processes [1]. But, it is not always easy to configure and use the developed systems. To improve the yield, the installation time must be the smallest to allow a fast profitability. So, the systems must not be only accessible to experts because of the setting complexity. The second encountered problem concerns the training samples set. Indeed, the customers do not provide lots of samples which are indispensable to train a supervised model. Thus, the output classes which define the training sample set are subjective, small and heterogeneous in terms of points per class. In the case of color matching, the subjectivity has an effect in the class definition, because the color perception is not the same for all people.

The aim of this paper herein is to present an original method to recognize the different output classes: colors (red,

Emmanuel Schmitt is with the Research Centre for Automatic Control, CRAN, France (email: emmanuel.schmitt@cran.uhp-nancy.fr).

Vincent Bombardier is with the Research Centre for Automatic Control, CRAN, CNRS, UMR 7039, Faculté des Sciences, Bd des Aiguillettes, BP 239, 54506 Vandoeuvre-lès-Nancy Cedex, France (phone: +33-3-83-68-4452; fax: +33-3-83-68-44-59; e-mail: vincent.bombardier@cran.uhpnancy.fr).

Patrick Charpentier is with the Research Centre for Automatic Control, CRAN, France (e-mail: patrick.charpentier@cran.uhp-nancy.fr). brown, white) or defects (knots, cracks, wanes). In the pattern recognition field, there exist lots of techniques to identify the objects characterized on the acquired images: Bayesian classifiers, non-Bayesian classifiers, neural networks, fuzzy logic classifiers ... In this study, the fuzzy logic seems to be appropriated to this kind of subjective problems. After giving the framework of these works, we justify and expose the chosen method based on fuzzy linguistic rules. Then, we show the generalization capabilities of our algorithm according to the number of training samples. Finally we essentially stress the simplification of the setting part in our method by explaining a self-fuzzification method based on the study of the output class typicality coefficients.

\section{FRAMEWORK}

This study takes place in a franco-luxembourger collaboration between the Research Centre for Automatic Control of Nancy and the Luxscan Technologies company. The main topic of these works is the development of a color matching vision system on wooden boards in order to improve the quality of manufactured products.

\section{A. Presentation of the production system}

The wooden board color recognition is made in real-time on an industrial environment. The used production lines can reach speeds of 220 meters of board length per minute. After the color identification, each piece of wood is sorted or cut according to the quality optimization criteria of the customers. Figure 1 illustrates an industrial process structure with its information flows.

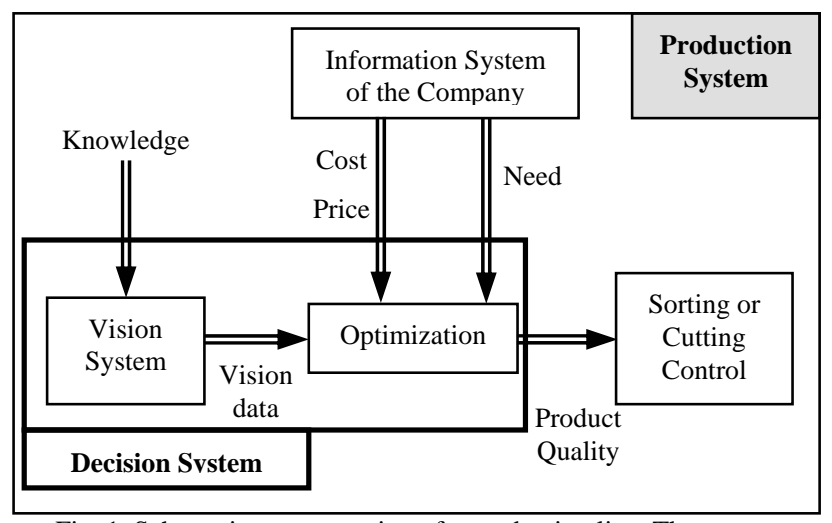

Fig. 1. Schematic representation of a production line. The arrows correspond to the information flows. 


\section{B. Presentation of the vision system}

The vision system is composed of color linear cameras which acquire the RGB components. These signals are sampled at $2 \mathrm{kHz}$. The measurements realized on the wooden boards are color images. These images are 24 bit RGB (Red, Green, Blue) images where each component takes a value between 0 and 255. In order to obtain a vision system nearest to human perception, the choice of another colorimetric reference space is necessary. The CIE (Commission Internationale de l'Eclairage) does not recommend the use of this RGB space. This space is non linear and its components are correlated. The information of luminance and chrominance are not separated. Thus, the CIE recommends the use of other spaces like HSI (HueSaturation-Intensity), Yuv, Luv, Lab. Several studies have been made on the colorimetric spaces [2][3]. Our choice concerns the Lab space, because it accurately retranscribes the colors seen by a human. After several tests on intra and interclass inertial values (Figure 2), we choose this colorimetric space which provides the better discrimination of the different color classes. The criteria of selection are the maximization of the equation (1) and the minimization of the equation (2).

Interclass inertia:

$$
I_{\text {interclass }}=\frac{1}{N} \sum_{k=1}^{K} n_{k} \cdot\left(d_{\text {eucl }}\left(C G_{N}, C G_{k}\right)\right)^{2}
$$

Intraclass inertia:

$$
I_{\text {intraclass }}=\frac{1}{N} \sum_{k=1}^{K} \sum_{i=1}^{n_{k}}\left(d_{\text {eucl }}\left(X_{k i}, C G_{k}\right)\right)^{2}
$$

with $\mathrm{N}$ the global point number,

$\mathrm{n}_{\mathrm{k}}$ the point number for the class $\mathrm{k}$,

$\mathrm{K}$ the class number,

$\mathrm{CG}_{\mathrm{N}}$ the gravity centre of the sample set,

$\mathrm{CG}_{\mathrm{k}}$ the gravity centre for the class $\mathrm{k}$,

$d_{\text {eucl }}(x, y)$ the Euclidian distance between the vectors $\mathrm{x}$ and $\mathrm{y}$,

$\mathrm{X}_{\mathrm{ki}}$ the $\mathrm{i}^{\text {th }}$ point of the class $\mathrm{k}$.

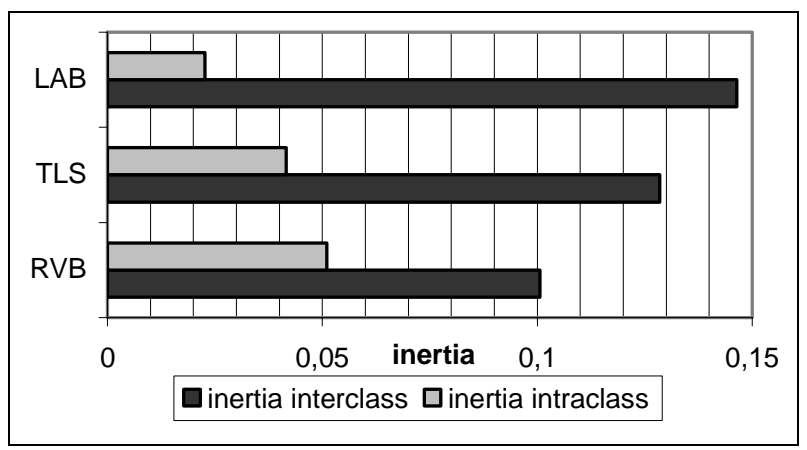

Fig. 2. Variation of the intra and interclass inertia coefficient for 3 colorimetric spaces.
To represent a color through the images, we have defined a simple characteristic vector from the industrial constraints (real-time aspect of the data processes). The data classically used are obtained from the color histograms [4][5]. The results of these studies are not reproducible in an industrial environment, because the colorimetric space is not chosen from its capability of discrimination. But, we retain the use of the proposed characteristic vector: the average of the different color components on a ROI (Region Of Interest). This choice is also justified by the simplicity of the calculations. Thus, it replies to the real-time constraints of the system. Several tests have allowed checking the good results obtained with this vector.

\section{PROPOSED METHOD OF CLASSIFICATION}

In the field of wooden board classification, several techniques are generally used. Some examples state the use of neural networks [6][7]. However, these methods have an important drawback during the step of model generation. The training sample number must be consistent in order that the neurons could focus on the wished output classes. In spite of the good results exposed in these articles, we focus on an original approach based on the fuzzy set theory [8][9]. Our choice is justified in two ways.

- The colors which must be classified are intrinsically fuzzy: impact of the wood grain in the wood color characterization, progressive transition between dark red and medium red in a same board for example. Thus, the extracted color descriptors are uncertain. So, the fuzzy logic allows working with this uncertainty.

- $\quad$ The customers express their needs under a nominal form with linguistic words. Thus, the output classes of the system are subjective and often not disjointed. There do not exist a strict boundary between medium brown and light brown in the color distribution. In this sense, we can say that the color perception is gradual.

In fact, the fuzzy logic concept is more adapted to the system which must reproduce the human reasoning [10]. To simplify the integration of the output lexical universe (output color classes defined by linguistic terms), we have chosen to use fuzzy linguistic rules [11]. The inference system used to identify the color is based on a fuzzy reasoning. The concepts introduced in this methodology are the elementary fuzzy proposition, the general fuzzy proposition, the fuzzy rules, the fuzzy inferences and the fuzzy relations [9].

There exist different kinds of rules which can define a fuzzy inference engine: the conjunctive rules and the implicative rules. These rules respectively gather, on the one hand, the possibility rules and the anti-gradual rules, on the other hand, the certainty rules and the gradual rules [11]. Two ways allow to obtain these rules; either from the expert knowledge (implicative rules), or from a training sample set 
(conjunctive rules). In our study, we generate the rules from numerical data, that's why we use conjunctive rules. This inference mechanism depends on a parallel activation of rules. Each rule provides a partial conclusion which is so aggregated to the others in the aim to give the final conclusion.

Our Fuzzy Reasoning Classifier (FRC) is based on an automatic generation of fuzzy rules according to a training sample set. The fuzzy inference engine follows a Larsen model because the Product operator is more adapted than the Minimum in the use of several premises [12]. Our rule generation part is based on the iterative form of the Ishibuchi-Nozaki-Tanaka's algorithm. It allows generating "IF... THEN..." fuzzy rules to define the different output classes. We distinguish three parts: the parameter fuzzification, the rules generation and the model adjustment [13]. Then the obtained rules are used to identify the different "non-labelled" samples. Figure 3 illustrates the principle of our FRC method.

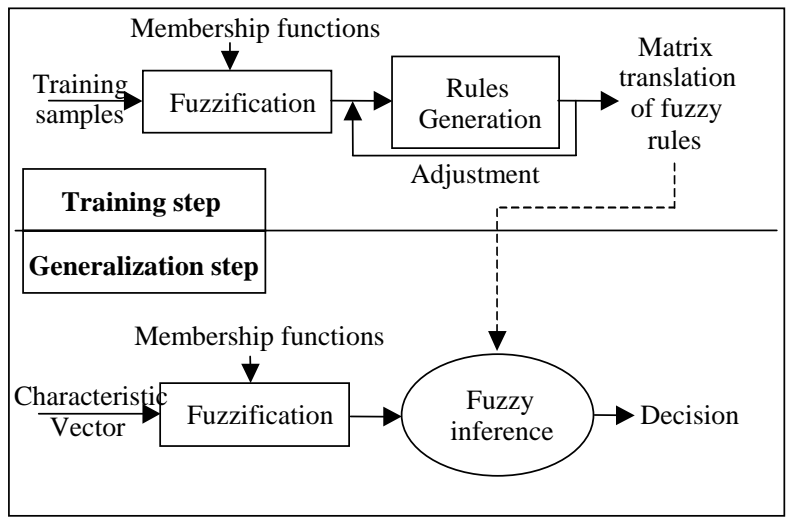

Fig. 3. Recognition module.

\section{GENERALIZATION CAPABILITIES OF THE FRC}

The training step is one of the stronger points of our method. Indeed, our project concerns a field in which the number of training data is small. It is possible that the output classes are represented with only ten or twenty samples. That's why, methods needing lots of points, like the neural networks, seem lesser adapted to our problem. We have realized a study concerning the impact of training sample number on the recognition rates. To make these tests, we use an industrial color data base which is composed of 900 points distributed in 6 color classes. Each class is represented by 150 samples. However, to evaluate and compare the different classifiers, points have been randomly generated by noising the real data with a white Gaussian noise. So, the final test base is composed of 5000 samples per class. Figure 4 represents the sample distribution in the La plan of the Lab colorimetric space.

We notice that there exist some confusion zones between the different color classes, and particularly between the lightest colors. After the comparison of classifiers, we will analyse the recognition rates per class.
The methods compared to the FRC algorithm are techniques frequently used in classification: Bayesian classifier, neural networks (NN), k nearest neighbour (kNN). Figure 5 illustrates the evolution of the recognition rates in generalization according to the size of the training sample number per class. The presented results are a mean value on several tests (30 attempts by training sample number).

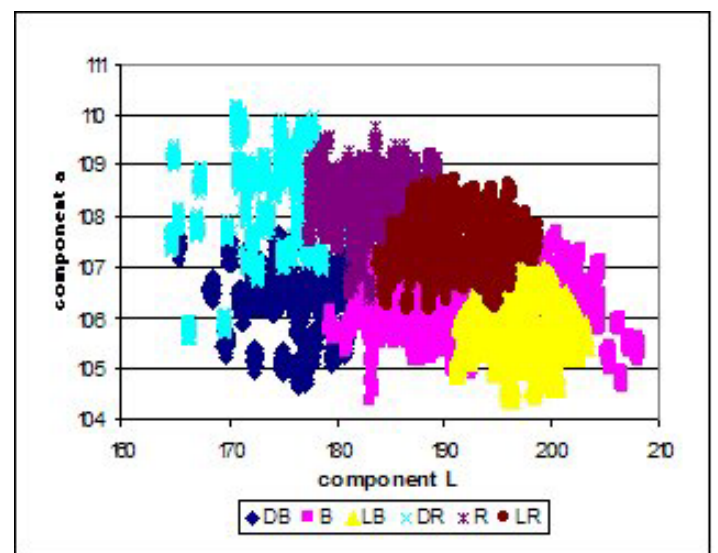

Fig. 4. Representation of the 6 artificial color classes in the La colorimetric space.

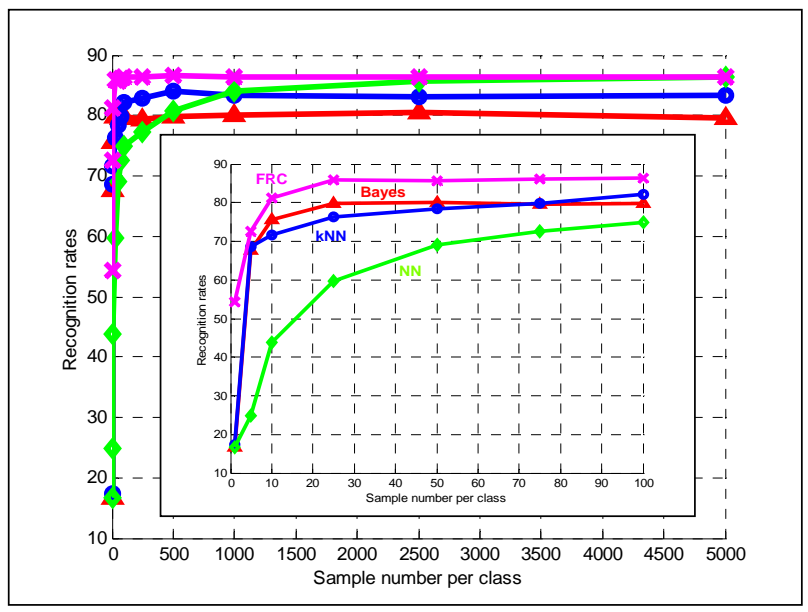

Fig. 5. Evolution of the recognition rates according to the training sample number per class.

We note that, the more there are points in the training sample set, the more the recognition rate increases. That is checked for all presented classification methods. An horizontal asymptote even appears: $80 \%$ for the Bayesian classifier, $83 \%$ for the $\mathrm{kNN}(\mathrm{k}=5), 86 \%$ for the $\mathrm{NN}, 86 \%$ for the FRC.

The different methods converge more or less quickly on these asymptotes. Typically, the neural networks need lots of samples during the training phase in order to be efficient in generalization. For equivalent performances, the neural networks need 40 times more points in the training step for the recognition rate to reach the one obtained with our method. This aspect is very important in our study, because it is very difficult to have big data sets in an industrial context. 
TABLE I

CONFUSION MATRIX FOR THE COLOR DATABASE IN GENERALIZATION - FRC

\begin{tabular}{|c|c|c|c|c|c|c|c|c|c|c|}
\hline & & \multicolumn{7}{|c|}{ F.R.C. Classification } & \multirow{2}{*}{$\begin{array}{c}\text { Number of } \\
\text { samples }\end{array}$} & \multirow{2}{*}{$\begin{array}{l}\text { Recognition } \\
\text { rates }\end{array}$} \\
\hline & & DB & $\mathrm{B}$ & LB & DR & $\mathrm{R}$ & LR & UK & & \\
\hline \multirow{7}{*}{ 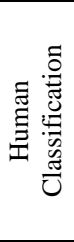 } & DB & 47 & 5 & 0 & 0 & 0 & 0 & 0 & 52 & $90.4 \%$ \\
\hline & B & 6 & 92 & 6 & 0 & 1 & 6 & 0 & 111 & $82.9 \%$ \\
\hline & LB & 0 & 6 & 167 & 0 & 0 & 5 & 0 & 178 & $93.8 \%$ \\
\hline & DR & 0 & 0 & 0 & 27 & 9 & 0 & 0 & 36 & $75 \%$ \\
\hline & $\mathrm{R}$ & 2 & 0 & 0 & 4 & 125 & 6 & 0 & 137 & $91.2 \%$ \\
\hline & LR & 0 & 3 & 15 & 0 & 15 & 83 & 0 & 116 & $71.6 \%$ \\
\hline & UK & 0 & 0 & 0 & 0 & 0 & 0 & $\mathbf{0}$ & $\mathbf{0}$ & $\mathbf{0 \%}$ \\
\hline \multicolumn{2}{|c|}{ Number of samples } & 55 & 106 & 188 & 31 & 150 & 100 & $\mathbf{0}$ & 630 & $85.87 \%$ \\
\hline
\end{tabular}

(DB: Dark Brown, MB: Medium Brown, LB: Light Brown, DR: Dark Red, MR: Medium Red, LR: Light Red, UK: Unknown)

In fact, the labelling is too drudgery and implies the intervention of qualified employees.

As said before, it is important to analyse the behaviour of our method for the classification of the different color classes. Indeed, these classes are heterogeneous in terms of sample quantity. Moreover, there exist some overlapping zones between the defined "customer" colors. Table I represents the confusion matrix corresponding to the recognition rate in generalization for a real color data set which contains 943 samples (313 for the training step and 630 for the generalization step). In this matrix, the number at the row $\boldsymbol{i}$ and the column $\boldsymbol{j}$ indicates the number of samples which are labelled in the class $\boldsymbol{i}$ by a human operator and recognized in the class $\boldsymbol{j}$ by the classification method. For instance, if we consider the DB class (Dark Brown), the classification module has recognized 47 samples in DB and 5 in the MB class (Medium Brown) whereas all these samples were labelled in the DB class by the human operator. From this matrix, it is possible to evaluate the acceptable confusions. The customer can accept confusions according to the intensity (Dark, Medium, Light), but he cannot accept hue variations (Red and Brown) which would downgrade the final product. For instance, if we consider the LR class (Light Red), we notice that 7 samples are recognized in the MR class (Medium Red). In this case, we can consider the classification as "right". However, there still remain 18 false classifications (15 in the class Light Brown and 3 in the class Medium Brown). In this case, if we accept the confusions, e.g. LR class recognized as MR class, the recognition rate is approximately increased by $10 \%$ to reach $94.6 \%$. The confusion matrix also allows the evaluation of the recognition model quality thanks to the calculation of the $\hat{\kappa}$ Kappa coefficient (6) [14]:

$$
\hat{\kappa}=\frac{N \sum_{i=1}^{r} X_{i i}-\sum_{i=1}^{r}\left(X_{i+} * X_{+i}\right)}{N^{2}-\sum_{i=1}^{r}\left(X_{i+} * X_{+i}\right)}
$$

where $\quad \mathrm{N}$ is the total number of samples $\mathrm{X}_{\mathrm{ii}}$ is the value in the row $\boldsymbol{i}$ and the column $\boldsymbol{i}$ $r$ is the number of output classes $\mathrm{X}_{\mathrm{i}+}$ is the sample number of the row $\boldsymbol{i}$
$\mathrm{X}_{+\mathrm{i}}$ is the sample number of the column $\boldsymbol{i}$.

In the presented case (Table. 1), this coefficient is equal to 0.82. According to the scale defined by Landis and Koch [14] our model is considered as excellent. Nevertheless, these results strongly depend on the fuzzification step [13].

\section{V.SELF-FUZZIFICATION OF THE INPUT PARAMETERS}

In an industrial environment, it is very difficult to employ experts in classification methods. So, it is important to simplify the setting step of our classification method and more precisely the fuzzification step. The main self-tuning methods are based on genetic algorithms [15] or clustering methods [16]. They are used to automatically tune the membership functions. However, the main drawback of technique based on genetic algorithms is the number of training samples per output class. Moreover, these methods directly work on the input data without taking into account the output classes and the expert needs.

\section{A. Presentation of the fuzzification}

The fuzzification consists in splitting in several terms the parameters which compose the characteristic vector. Each decomposition or term gets closer to a word of the natural language. For instance, the luminance can be "light", "medium" or "dark". There exit two ways to set the fuzzification: a fuzzification with an equal distribution of the terms, and a fuzzification with a repartition adapted to the training data set.

There are two parts of the fuzzification step which can have an impact on the recognition rates: the number of terms and the fuzzification curve form. Usually, the number of terms is empirically chosen. Figure 6 illustrates an example of 3-term decompositions. We will present in the section IV.C a set of results concerning the impact of the fuzzification on the recognition rates.

\section{B. Analysis of the parameter typicalities}

The users of our method prefer to quickly configure the parameter space decomposition, because they do not have the sufficient knowledge of the classification method. Thus, they define an equal distributed fuzzification. However, if the splitting does not correspond to the variation space of the 
real data set, the defined terms can be inappropriate. We can say that the data are not typical of the obtained subspaces. We propose to base our self-fuzzification method on the typicality of the characteristic vector [17]. This notion is evaluated with the calculation of the typicality coefficient for the different parameters. The typicality calculation (5) is based on the intern dissimilarity (3) and extern likeness (4) evaluations for all samples.

$$
\begin{aligned}
& R\left(V_{a}^{u}\right)=\frac{\sum_{i=1}^{n} \frac{1}{d_{\text {eucl }}\left(V_{a}^{u}, V_{a}^{f_{i}}\right)}}{n} \\
& D\left(V_{a}^{u}\right)=\frac{\sum_{i=1}^{m} \frac{1}{1-d_{\text {eucl }}\left(V_{a}^{u}, V_{a}^{e_{i}}\right)}}{m} \\
& T\left(V_{a}^{u}\right)=\frac{R \cdot D}{R \cdot D+(1-R) \cdot(1-D)}
\end{aligned}
$$

where $V_{a}^{u}$ is the value of the parameter a for the point u;

$V_{a}^{f_{i}}$ is the value of the parameter a for the "friend" point $\mathrm{f}$ of the class $\mathrm{i}$;

$V_{a}^{e_{i}}$ is the value of the parameter a for the "enemy" point e of the class i; $d_{\text {eucl }}(x, y)$ is the Euclidian distance between $\mathrm{x}$ and $\mathrm{y}$;

$\mathrm{n}$ is the number of "friend" points;

$\mathrm{m}$ is the number of "enemy" points;

$\mathrm{R}$ and $\mathrm{D}$ correspond to $R\left(V_{a}^{u}\right)$ and $D\left(V_{a}^{u}\right)$.

Figure 6 represents the variation of the typicality coefficients for our industrial color data base. There are 6 wooden color classes: Dark Brown (DB), Medium Brown (B), Light Brown (LB), Dark Red (DR), Medium Red (R) and Light Red (LR).

From the typicality coefficients, we have evaluated the ratio $\rho_{\text {corr/xcorr }}$ between the correlation coefficient and the cross-correlation for the different output classes. Then, these coefficients allow defining the fuzzification terms from the maximum value of the ratio. If two classes are successive and if the ratio between these two classes is the maximum value, we consider that these classes could be represented by the same term.

Algorithm:

1) Calculation of $\rho_{\text {corr/xcorr }}$ ratio for all classes

2) Ascending class sorting from the parameter values

\begin{tabular}{|c|c|c|c|c|c|c|}
\cline { 2 - 7 } \multicolumn{1}{c|}{ Ex : } & $\mathrm{DR}$ & $\mathrm{DB}$ & $\mathrm{R}$ & $\mathrm{B}$ & $\mathrm{LR}$ & $\mathrm{LB}$ \\
\hline class & $\mathrm{C}_{1}$ & $\mathrm{C}_{2}$ & $\mathrm{C}_{3}$ & $\mathrm{C}_{4}$ & $\mathrm{C}_{5}$ & $\mathrm{C}_{6}$ \\
\hline
\end{tabular}

3) For $i=C_{1}$ to $C_{K}$,

if $\rho_{\text {corr/xcorr }}\left(C_{i}, C_{i+1}\right)=\max \left[\rho_{\text {corr/xcorr }}\left(C_{i}, C_{j}\right) \mid j \in[1, K], j \neq i\right]$ then $C_{i}$ and $C_{i+1}$ are represented with the same term else the term only represents the class $C_{i}$, and another term is created for the class $\mathrm{C}_{\mathrm{i}+1}$.

By applying this methodology on the previous example (Figure 6), the parameter $\mathrm{L}$ has been fuzzified in 3 terms.

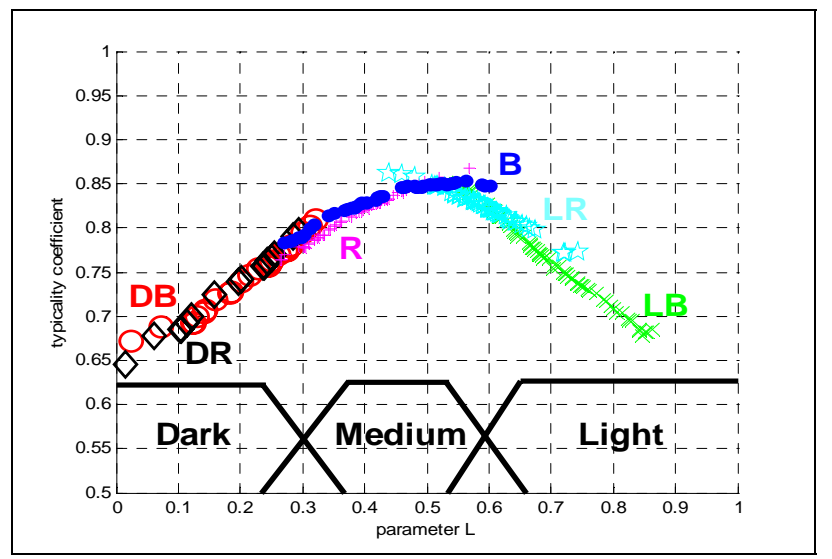

Fig. 6. Typicality coefficient and fuzzification curves (bold line) for the luminance.

\section{Fuzzification impact on the recognition rates}

As said in the section IV.A, the fuzzification has a direct impact on the recognition rates. To study this effect, we use the industrial color data base labeled by a customer presented in the IV section. The 943 samples are distributed in the previously 6 classes. For each class, one training set and one checking set have been built. Table II present the generalization recognition rates according to the fuzzification curve form and the number of terms, in the case of an equal distribution.

TABLE II

RECOGNITION RATES IN GENERALIZATION EQUAL DISTRIBUTED FUZZIFICATION

\begin{tabular}{c|c|c|c|c}
\hline \hline $\begin{array}{c}\text { Numbers of } \\
\text { fuzzification } \\
\text { terms }\end{array}$ & $\begin{array}{c}\text { Gaussian } \\
\text { curves }\end{array}$ & $\begin{array}{c}\text { Triangular } \\
\text { curves }\end{array}$ & $\begin{array}{c}\text { Trapezoidal } \\
\text { curves }\end{array}$ & $\begin{array}{c}\text { Trapezoidal } \\
\text { Triangular } \\
\text { curves }\end{array}$ \\
\hline 2 & $46.4 \%$ & $48.2 \%$ & $49.8 \%$ & $49.8 \%$ \\
3 & $55.1 \%$ & $69.8 \%$ & $74.3 \%$ & $77.0 \%$ \\
5 & $69.9 \%$ & $75.5 \%$ & $78.6 \%$ & $83.7 \%$ \\
7 & $73.4 \%$ & $\mathbf{8 0 . 0} \%$ & $\mathbf{8 1 . 8 \%}$ & $\mathbf{8 5 . 9 \%}$ \\
9 & $71.6 \%$ & $74.3 \%$ & $79.6 \%$ & $82.1 \%$ \\
11 & $71.0 \%$ & $72.9 \%$ & $76.1 \%$ & $79.6 \%$ \\
\hline \hline
\end{tabular}

The best results are obtained for a 7-term equal distributed fuzzification with Trapezoidal-Triangular curves. If the parameter representation space is too much split, the recognition rates decrease because the color classes are not represented by the subspace of each term. In fact, there are more confusion zones in the representation space. Then, we apply our self-fuzzification method on the same data base. Table III illustrates a comparison between the better equal distributed fuzzification, a manually adapted fuzzification and the self-fuzzifications. For these tests, the fuzzification curves are trapezoidal-triangular. Moreover, we compare the FRC results with the current industrial classifier. 
TABLE III

EQUAL DISTRIBUTED FUZZIFICATION VERSUS ADAPTED FUZZIFICATION - COMPARISON WITH THE CURRENT INDUSTRIAL CLASSIFIER (1 NEAREST NEIGHBOUR)

\begin{tabular}{|c|c|c|c|c|c|c|c|c|c|}
\hline & \multirow{2}{*}{$\begin{array}{c}\begin{array}{c}\text { Industrial } \\
\text { classifier }\end{array} \\
\text { Rates }\end{array}$} & \multirow{2}{*}{$\begin{array}{c}\begin{array}{c}\text { Bayesian } \\
\text { Classifier }\end{array} \\
\text { Rates }\end{array}$} & \multirow{2}{*}{$\begin{array}{c}\begin{array}{c}\text { Neural } \\
\text { Networks }\end{array} \\
\text { Rates }\end{array}$} & \multicolumn{2}{|c|}{$\begin{array}{l}\text { 7-equal distributed } \\
\text { fuzzification - FRC }\end{array}$} & \multicolumn{2}{|c|}{$\begin{array}{c}\text { Manually adapted } \\
\text { Fuzzification - FRC } \\
\end{array}$} & \multicolumn{2}{|c|}{ Self-fuzzification - FRC } \\
\hline & & & & Rates & $\begin{array}{l}\text { Number of } \\
\text { rules }\end{array}$ & Rates & $\begin{array}{l}\text { Number of } \\
\text { rules }\end{array}$ & Rates & $\begin{array}{c}\text { Number of } \\
\text { rules }\end{array}$ \\
\hline Data Set $1^{*}$ & $81.37 \%$ & $79.12 \%$ & $73.92 \%$ & $85.87 \%$ & 343 & $84.28 \%$ & 75 & $87.14 \%$ & 24 \\
\hline Data Set $2^{* *}$ & $80.29 \%$ & $78.85 \%$ & $71.25 \%$ & $84.92 \%$ & 343 & $83.51 \%$ & 75 & $86.10 \%$ & 45 \\
\hline
\end{tabular}

* Data Set 1 is composed of 943 samples distributed in 6 classes (313 for the training step and 630 for the generalization step)

** Data Set 2 is composed of 1314 samples distributed in 6 classes (209 for the training step and 1105 for the generalization step)

Two aspects are interesting: the recognition rates and the number of generated fuzzy rules. When the fuzzification is adapted to the training data, the number of rules is smaller than the one obtained with an equal distribution. Moreover, in our case, the fuzzification based on expert knowledge [18] doesn't really provide better classification rates, even if it decreases the number of rules. Our fuzzification method combines both advantages. Indeed, by decreasing the rule number and in the same way the consumed time for classification, the recognition rate increases by $1.2 \%$. Our self-fuzzification method have been compared to others methods like clustering [16] and genetic algorithms [15], but the results are worse than those obtained with our proposition. (approximately $2 \%$ ).

\section{Conclusions}

In this article, we have presented a method allowing a fuzzy linguistic rule classification from a reduced training data set. Moreover, a technique of self-fuzzification has been proposed to reduce the difficulties of the system settings. The aim of this method is double. Firstly, having few samples at our disposal to generate the numerical model, we must find a method with a strong capability of generalization. This study realized on color data clearly shows this capability of our method. Indeed, with only 25 training samples per class, we obtain the better recognition rates. Moreover, even if the output classes present overlapping zones, our methodology does not perform many misclassifications. Secondly, by using a self-fuzzification based on the typicality coefficient analysis, our methodology provides better results than an equal-distributed fuzzification. The typicality has the advantage to represent the common points between the different output classes. Our fuzzification method allows obtaining a fuzzification based on the customer's specified output classes. In addition to the recognition rate improvement, the effective classification time is reduced thanks to few generated fuzzy rules. To improve our classification method, it is possible to extend our self-fuzzification to a technique of knowledge integration in the model generation [18].

\section{ACKNOWLEDGMENT}

The authors wish to thank their industrial partner the LuxScan Technologies Company who follows and supports the present works.

\section{REFERENCES}

[1] A. H. Huber, S. Ruddell and C. W. McMillin, "Industry standards for recognition of marginal wood defects," Forest Products Journal, vol. 40(3), pp. 30-34, 1990.

[2] I. Philipp and T. Rath, "Improving plant discrimination in image processing by use of different colour space transformations", Computers and electronics in agriculture, vol. 35, pp. 1-15, 2002.

[3] K. Leon, D. Mery, F. Pedreschi and J. Leon, "Color measurement in L*a*b* units from RGB digital images,” Food research international, vol. 39, pp. 1084-1091, 2006.

[4] Q. Lu, A Real-Time System for Color Sorting Edge-Glued Panel Parts, Ph.D. Dissertation, Faculty of the Virginia Polytechnic Institute and State University, Blacksburg, Virginia, 1997.

[5] A. Hanbury, Mathematical morphology on the Unit Circle with applications to the hues and the oriented textures (in French), Ph.D. Dissertation, Ecole Nationale Supérieure des Mines, Paris, 2002.

[6] D. T. Pham and S. Sagiroglu, "Training multilayered perceptrons for pattern recognition: a comparative study of four training algorithms," International Journal of Machine Tools and Manufacture, vol. 41, pp. 419-430, 2001.

[7] P. A. Estevez, C. A. Perez and E. Goles, "Genetic input selection to a neural classifier for defect classification of radiata pine boards," Forest Products Journal, vol. 53, pp. 87-94, 2003.

[8] L. A. Zadeh, "Fuzzy sets,” Information and control, vol. 8, pp. 338353, 1965.

[9] B. Bouchon-Meunier, The fuzzy logic and its applications (in French), Edited by Addison-Wesley, 1995.

[10] L. A. Zadeh, "Outline of a new approach to the analysis of complex systems and decision processes," IEEE Transactions on Systems, Man, and Cybernetics, vol. SMC-3, pp. 28-44, 1973.

[11] D. Dubois and H. Prade, "What are Fuzzy rules and how to use them?,” Fuzzy Sets and Systems, vol. 84, pp. 169-185, 1996.

[12] H. Ishibuchi, K. Nozaki and H. Tanaka, "Distributed representation of fuzzy rules and its application to pattern classification,” Fuzzy Sets and Systems, vol. 52, pp. 21-32, 1992.

[13] E. Schmitt, C. Mazaud, V. Bombardier and P. Lhoste, “A Fuzzy Reasoning Classification Method for Pattern Recognition,” Fuzzy IEEE, Vancouver, Canada, 2006.

[14] J. R. Landis and G. G. Koch, "The measurement of observer agreement for categorical data,” Biometrics, vol. 33, pp. 159-174, 1977.

[15] O. Cordon, F. Gomide, F. Herrera, F. Hoffmann and L. Magdalena, "Ten years of genetic fuzzy systems: current framework and new trends”, Fuzzy Sets and Systems, vol. 141, pp. 5-31, 2004.

[16] F. A. T. De Carvalho, "Fuzzy c-means clustering methods for symbolic interval data”, Pattern Recognition Letters, vol. 28, pp. 423437, 2007.

[17] J. Forest, M. Rifqi and B. Bouchon-Meunier, “Class Segmentation to Improve Fuzzy Prototype Construction: Visualization and Characterization of Non Homogeneous Classes (in French)," Rencontres Francophones sur la Logique Floue et ses Applications LFA, Toulouse, France, pp. 29-36, 2006.

[18] V. Bombardier, C. Mazaud, P. Lhoste and R. Vogrig, "Contribution of fuzzy reasoning method to knowledge integration in a defect recognition system,” Computer in Industry, In Press, 2006. 